\title{
String Breaking and Quarkonium Dissociation at Finite Temperatures
}

\author{
S. Digal, P. Petreczky and H. Satz \\ Fakultät für Physik, Universität Bielefeld \\ D-33501 Bielefeld, Germany
}

\begin{abstract}
:
Recent lattice studies of string breaking in QCD with dynamical quarks determine the in-medium temperature dependence of the heavy quark potential. Comparing this to the binding energies of different quarkonium states, we check if these can decay into open charm/beauty in a confined hadronic medium. Our studies indicate in particular that the $\chi_{c}$ and the $\psi^{\prime}$ dissociate into open charm below the deconfinement point.
\end{abstract}

The behavior of quarkonium states in a hot strongly interacting medium was proposed as test for its confinement status [1]. In sufficiently hot deconfined matter, color screening will dissolve the binding of the heavy quark-antiquark pair; hence the higher excited states with smaller binding energies and larger radii will break up at lower temperatures. On a microscopic level, it was argued that only a hot deconfined medium provides sufficiently hard gluons to dissociate the tighly bound quarkonium states [2]; this again leads to a dissociation hierarchy as function of the binding energy. However, the arguments depend on a large binding energy, and it is not clear to what extent they remain valid for higher excited states. In particular, the $\psi^{\prime}$ has a mass only $50 \mathrm{MeV}$ below the open charm threshold, to that it should be readily dissociated in any (confined or deconfined) medium. It is thus of considerable interest to study possible collective mechanisms for quarkonium dissociation in a confined medium.

The study of string breaking at finite temperatures provides a new way to address this problem. String breaking will presumably occur when the string potential reaches twice the mass of the lowest light-heavy meson [3]. Hence if a charmonium state, stable under strong interactions at $T=0$, is to be broken up in a confining environment with $T>0$, the gap between the mass of the state and the open charm threshold has to be overcome, so that the decay into a $D^{+} D^{-}$pair becomes energetically favorable. This can occur if the mass of the open charm meson decreases with temperature faster than the charmonium mass. Lattice studies of the heavy quark potential [4, 5] now determine the temperature dependence of the string breaking threshold. The investigation of the suppression of charmonium production below the deconfinement point thus becomes possible; the same arguments apply of course to bottomonium production as well. The aim of this paper is 
to study such hadronic in-medium decay dissociation of charmonium and bottomonium states.

In the absence of any medium, charmonium and bottomonium spectra are quite well described by non-relativistic potential theory [6]-[8]. The basis for this is given by the Schrödinger equation

$$
\left[2 m_{a}+\frac{1}{m_{a}} \nabla^{2}+V_{1}(r)\right] \Phi_{i}^{a}=M_{i}^{a} \Phi_{i}^{a}
$$

where $a=c, b$ specifies charm or bottom quarks, $i$ denotes the quarkonium state in question, and the reduced radius $r$ is the separation of the two heavy quarks. The confining color singlet potential can be parametrized as

$$
V_{1}(r)=\sigma r-\frac{\alpha}{r}
$$

in terms of the string tension $\sigma$ and a $1 / r$ contribution containing both Coulombic and transverse string effects [6]; for other forms, see [8]. It is evident that Eq. (2) is a 'quenched' idealization, since in full QCD the string connecting the two heavy quarks will break when its energy surpasses that of two dressed light quarks. Hence a more realistic form would be

$$
\begin{aligned}
V_{1}(r) & =\sigma r-\frac{\alpha}{r} & & \forall r \lesssim r_{h}, \\
& =E_{s} & & \forall r \gtrsim r_{h},
\end{aligned}
$$

where $r_{h} \simeq E_{s} / \sigma$ indicates the typical hadronic confinement scale and $E_{s}$ the energy needed to break the string between the heavy quarks. Since this energy is related to the energy required to bring a virtual light $q \bar{q}$ pair on-shell as a hadron, we expect it to be related to a chiral symmetry breaking constituent quark mass, independent of the masses of the heavy quarks. From Eq. (11), the energy needed to break up quarkonium state $(a, i)$ is

$$
E_{a, i}=2 m_{a}+E_{s}^{a}-M_{i}^{a} .
$$

To achieve a break-up, we have to overcome the energy difference between the quarkonium state and two open charm or beauty mesons, so that

$$
E_{c, i}=2 M_{D}-M_{i}^{c} \quad, \quad E_{b, i}=2 M_{B}-M_{i}^{b},
$$

with $M_{D}$ and $M_{B}$ denoting the lightest open charm and beauty mesons, respectively. Using $m_{c} \simeq 1.3 \mathrm{GeV}$ and $m_{b} \simeq 4.7 \mathrm{GeV}$ as indicative heavy quark mass values [0], 9], we get from these two equations

$$
E_{s}^{c}=2\left(M_{D}-m_{c}\right) \simeq 1.1 \mathrm{GeV}
$$

and

$$
E_{s}^{b}=2\left(M_{B}-m_{b}\right) \simeq 1.1 \mathrm{GeV},
$$

for $c \bar{c}$ and $b \bar{b}$ states, respectively. The fact that the two values agree is reassuring in two ways: first, it indicates that indeed the chiral properties of the QCD vacuum determine $E_{s}$, not the masses of the heavy quarks. Secondly, with $\sigma \simeq 0.19 \mathrm{GeV}^{2}[7$, 9], the break-up occurs for $r_{h} \simeq 1 / \sigma \simeq 1 \mathrm{fm}$, in accord with the expected range of confining forces. 
In the presence of a medium, we expect the break-up to become easier with increasing temperature, since presumably the effective constituent quark mass as well as the string tension will decrease. Previously [10, 11], the evolution of quarkonium binding with temperature was studied on the basis of a screened form of the Cornell potential (2); the dissociation values of the screening mass, defined by the divergence points of the corresponding bound state radii, were then related to the temperature through quenched finite temperature lattice results. Such a procedure can at best give some first idea of the dissociation pattern; in particular, it can only give upper limits for the existence of higher excited states. For a more realistic treatment, the heavy quark potential must be determined directly in lattice studies of full QCD. This will then not only allow us to study the effect of color screening in a deconfined medium, but also the evolution of the gap between the bound state mass and the open charm/beauty threshold. Such studies will indicate if and for what states the dissociation can occur already in a confined medium and at the same time provide the associated dissociation temperatures.

In finite temperature lattice QCD, the temperature behavior of the heavy quark potential $V(T, r)$ is obtained from Polyakov loop correlations,

$$
-T \ln <L(0) L^{+}(r)>=V(T, r)+C .
$$

The constant $C$ includes both the cut-off dependent self-energy and the entropy contributions $-T S$ (for $T \neq 0,-T \ln <L(0) L^{+}(r)>$ is actually the free energy of the $Q \bar{Q}$ pair and not the static energy, as in the zero temperature case [5]). The constant $C$ can be fixed by requiring that at very short distances, $r<<T^{-1}$, the potential has the zero temperature form, since in the limit $r \rightarrow 0$, the effects of the medium should become negligible. Present lattice calculations are perhaps not yet precise enough to reach sufficiently small $r$; nevertheless, they should provide some reasonable indication of the behavior of the potential.

The free energy (8) was recently studied in considerable detail on $16^{3} \times 4$ lattices, for three species of light quarks, using an improved gauge and staggered fermion action [5]. Some representative temperature curves of the resulting potential in units of $\sqrt{\sigma}(8)$ are shown in Fig. 1. The normalization constant $C$ was fixed here by requiring the potentials to agree with the zero temperature Cornell potential (2) at $r=1 /(4 T)$. For this we have taken $\alpha=0.4$ in Eq. (2), which is the value estimated in 3-flavor QCD [9]; the value of $\sigma$ is irrelevant if $V(r, T)$ and $r$ are measured in units of $\sqrt{\sigma}$. It is seen that beyond a certain separation distance, the potential of the heavy quark system reaches a constant value $V_{\infty}(T)$, corresponding to string breaking and hence the formation of an "open charm" $Q \bar{q}-\bar{Q} q$ pair. The results shown in Fig. 11 were obtained for quark mass $m_{q}=0.4 T$; however, a more detailed study of the quark mass dependence shows that this value is already very close to the chiral limit [5]. The lattice results thus provide a reasonable estimate of the temperature behavior of $V_{\infty}(T)$. It is shown in Fig. 2, including the value $V_{\infty}(0) \simeq E_{s} \simeq 1.1 \mathrm{GeV}$ from Eqs. (6.7). We note that in the vicinity of the deconfinement point $T=T_{c}$, the potential drops quite sharply, as expected.

As mentioned, the potential form shown in Figs. 1 and 2 was obtained by normalizing the lattice results to the zero temperature Cornell potential (2) at the reference point $r=$ $1 /(4 T)$; the present lattice size does not permit smaller $r$. For $T=T_{c}$, the normalization point corresponds to $r \simeq 0.3 \mathrm{fm}$; however, then deviations from the Cornell potential set in at essentially this distance, so that for $T \rightarrow T_{c}$, the normalization becomes unreliable. 
In general, medium effects, such as a temperature dependence of the string tension $\sigma$, may begin to affect the static potential at this distance, and as a consequence, also the masses of quarkonia states may become temperature-dependent [12]. To check the potential form used for normalization, we therefore want to consider a temperature-dependent string potential instead of Eq. (2). Such a potential was found to describe quenched lattice results on the heavy-quark potential for $T<T_{c}$ quite well, if it has the form [13, 14]

$$
\begin{aligned}
V_{\text {string }}(r, T)= & \left(\alpha-\frac{1}{6} \arctan (2 r T)\right) \frac{1}{r}+ \\
& \left(\sigma(T)-\frac{\pi T^{2}}{3}-\frac{2 T^{2}}{3} \arctan \frac{1}{r T}\right) r+ \\
& \frac{1}{2} \ln \left(1+4 r^{2} T^{2}\right) .
\end{aligned}
$$

Normalizing the Polyakov loop correlator (8) at $r=1 /(4 T)$ with Eq. (9), we thus obtain what might be a more reliable estimate of the plateau $V_{\infty}(T)$ than with the $T=0$ form (2). In Eq. (9), we again set $\alpha=0.4$ [9] and take $\sigma(T)$ as given in [14. It turns out, however, that the two forms of short distance behavior resulting from Eqs. (2) and (9) are practically identical, so that the normalisation is in fact not affected by the in-medium modifications at larger distances.

To consider further possible uncertainties of the normalisation procedure, we have also normalized the Polyakov loop correlator at the next smallest distance $r=\sqrt{2} /(4 T)$. The resulting two forms of $V_{\infty}(T)$ are shown in both Fig. 3 and Fig. 4 . The difference between the two curves of $V_{\infty}(T)$ provides an estimate of the normalization error. Except for the region very near $T=T_{c}$, the uncertainty is seen to be quite small.

Next, we then estimate the quarkonium masses based on (9). In the zero temperature limit, Eq. (9) reduces to Eq. (2); this describes the quarkonium results quite well, if relativistic corrections to the potential are taken into account [9]. The temperature dependence of the relativistic correction to the static potential is not known. For bottomonium, the relativistic corrections are small; for charmonium, they may have to be included. On the other hand, we have found that a reasonably good description can be obtained with the c-quark mass $m_{c}=1.3 \mathrm{GeV}$ (in [9], the value 1.38 was used). Thus zero temperature quarkonium spectroscopy can be reproduced quite well with $\alpha=0.4$, $\sqrt{\sigma}=0.44 \mathrm{GeV}, m_{b}=4.72$ and $m_{c}=1.3 \mathrm{GeV}$. These values were used to predict the finite temperature quarkonium masses in our present study. In Table 1 we list all states, together with the corresponding zero temperature dissociation energies, and in Figs. 3 and Ð we include the resulting temperature dependence of the quarkonium masses, in order to determine their survival patterns. It is seen that $J / \psi, \Upsilon$, and $\chi_{b}$ states are likely to survive up to temperatures $T \geq T_{c}$ and thus are out of the region we are able to consider here. The $\Upsilon$ ' state dissociates in a temperature region in which the value of the open beauty threshold suffers from large uncertainties in the normalization of the Polyakov loop correlator. Thus for this state we can only give an lower limit for the dissociation. On the other hand, the $\psi^{\prime}, \chi_{c}, \chi_{b}^{\prime}$ and $\Upsilon$ " masses intersect the string breaking curve noticably below $T_{c}$; the intersection values are listed in Table 1 . Their dissociation occurs through hadronic decay, made possible because in-medium effects have effectively reduced the constituent quark mass enough to lower the open charm or beauty threshold below the mass values of the respective states. Hence they simply break up into open charm or 
beauty mesons; this is not a signal for deconfinement, but rather indicates the approach of chiral symmetry restoration and the resulting decrease of the constituent quark mass. To probe the medium in a deconfined state, we have to consider the more tightly bound states $J / \psi, \chi_{b}$ and $\Upsilon$.

Evidently these conclusions differ from considerations of $\chi_{c}$ dissociation as possible signal for the onset of deconfinement [15]. It should be noted, however, that previous screening studies [11] really could conclude only that $\psi^{\prime}$ and $\chi_{c}$ cannot exist for $T \geq T_{c}$; a prior in-medium dissociation of the type obtained here was not excluded, but it could be addressed only once the temperature dependence of the heavy quark potential and thus of string breaking became accessible.

\begin{tabular}{|c||c|c|c||c|c|c|c|c|}
\hline state & $J / \psi$ & $\chi_{c}$ & $\psi^{\prime}$ & $\Upsilon$ & $\chi_{b}$ & $\Upsilon^{\prime}$ & $\chi_{b}^{\prime}$ & $\Upsilon^{\prime \prime}$ \\
\hline \hline$E_{s}^{i}[\mathrm{GeV}]$ & 0.64 & 0.20 & 0.05 & 1.10 & 0.67 & 0.54 & 0.31 & 0.20 \\
\hline \hline$T_{d} / T_{c}$ & - & 0.74 & $0.1-0.2$ & - & - & $\gtrsim 0.93$ & 0.83 & 0.74 \\
\hline
\end{tabular}

Table 1: Quarkonium Dissociation by String Breaking

In summary, lattice studies of the heavy quark potential in QCD with dynamical quarks indicate that there are two collective forms for quarkonium dissociation in hot matter. For $T<T_{c}$, hadronic in-medium effects, related to the approach to chiral symmetry restoration and to the decrease of the string tension, lead to a reduction of the string-breaking threshold. This in turn results in the dissociation of the higher excited quarkonium states $\left(\psi^{\prime}, \chi_{c}, \chi_{b}^{\prime}, \Upsilon^{\prime \prime}\right)$ through decay into open charm or beauty. The more tightly bound lower states $\left(J / \psi, \Upsilon, \chi_{b}\right.$ and perhaps also the $\left.\Upsilon^{\prime}\right)$ survive up to or beyond $T_{c}$. Their dissociation will thus occur through color screening in a deconfined medium; the analysis of this is carried out in [16], based on the corresponding QCD lattice studies for $T \geq T_{c}$ [5].

\section{Acknowledgements}

It is a pleasure to thank F. Karsch and E. Laermann for numerous helpful discussions. The financial support from DFG under grant Ka 1198/4-1 and from BMFB under grant 06 BI 902 is gratefully acknowledged.

\section{References}

[1] T. Matsui and H. Satz, Phys. Lett. B 178 (1986) 416.

[2] D. Kharzeev and H. Satz, Phys. Lett. B 334 (1994) 155.

[3] O. Philipsen and H. Wittig, Phys. Rev. Lett. 81 (1998) 4056. 
[4] C. DeTar et al., Phys. Rev. D 59 (1999) 03150.

[5] F. Karsch, E. Laermann and A. Peikert, hep-lat/0012023.

[6] E. Eichten et al., Phys. Rev. D 17 (1978) 3090; Phys. Rev. D 21 (1980) 203.

[7] S. Jacobs, M. G. Olsen and C. Suchyta III, Phys. Rev. D 33 (1986) 3338.

[8] E. Eichten and C. Quigg, Phys. Rev. D 52 (1995) 1726.

[9] G. Bali, K. Schilling and A. Wachter, Phys. Rev. D 56 (1997) 2566.

[10] F. Karsch, M. T. Mehr and H. Satz, Z. Phys. C 37 (1988) 617.

[11] F. Karsch and H. Satz, Z. Phys. C 51 (1991) 209.

[12] R. Vogt and A. Jackson, Phys. Lett. B 206 (1988) 333.

[13] M. Gao, Phys. Rev. D 40 (1989) 2708.

[14] O. Kaczmarek et al., Phys. Rev. D 62 (2000) 034021.

[15] See e.g., H. Satz, Rep. Prog. Phys. 63 (2000) 1511, and further references there.

[16] S. Digal, P. Petreczky and H. Satz, to appear. 


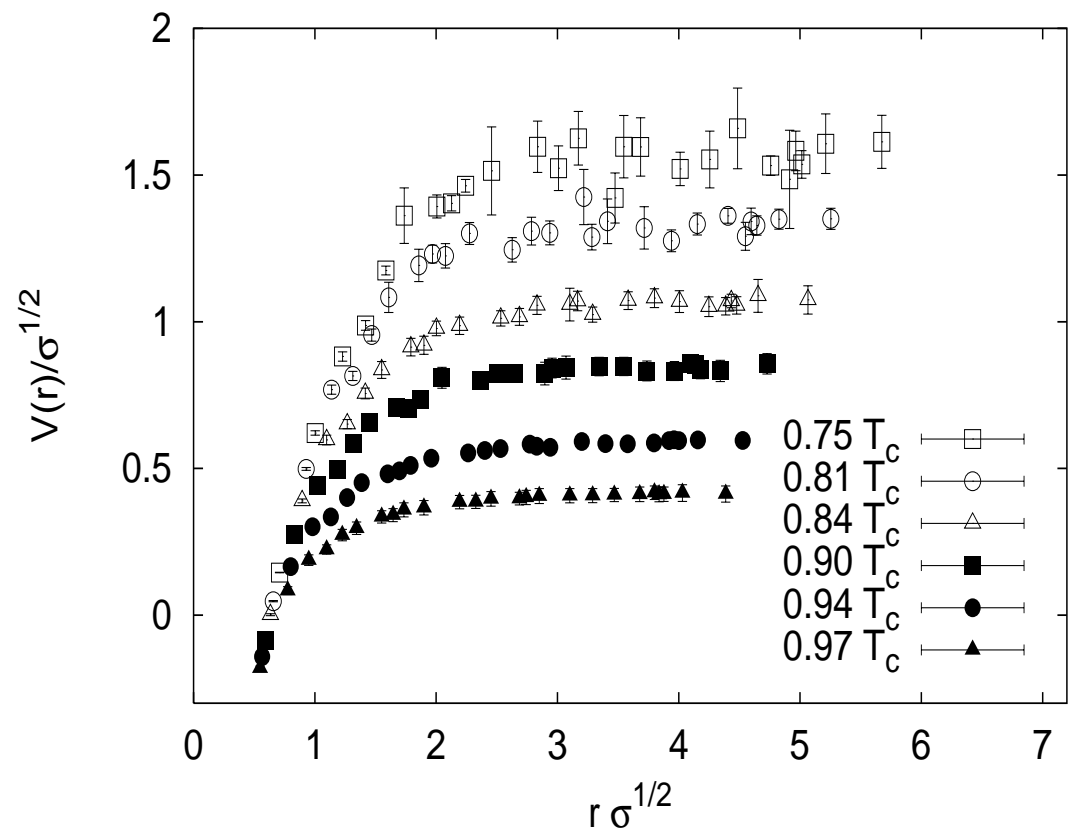

Figure 1: The heavy quark potential at different temperatures, normalized to the Cornell potential at short distance.

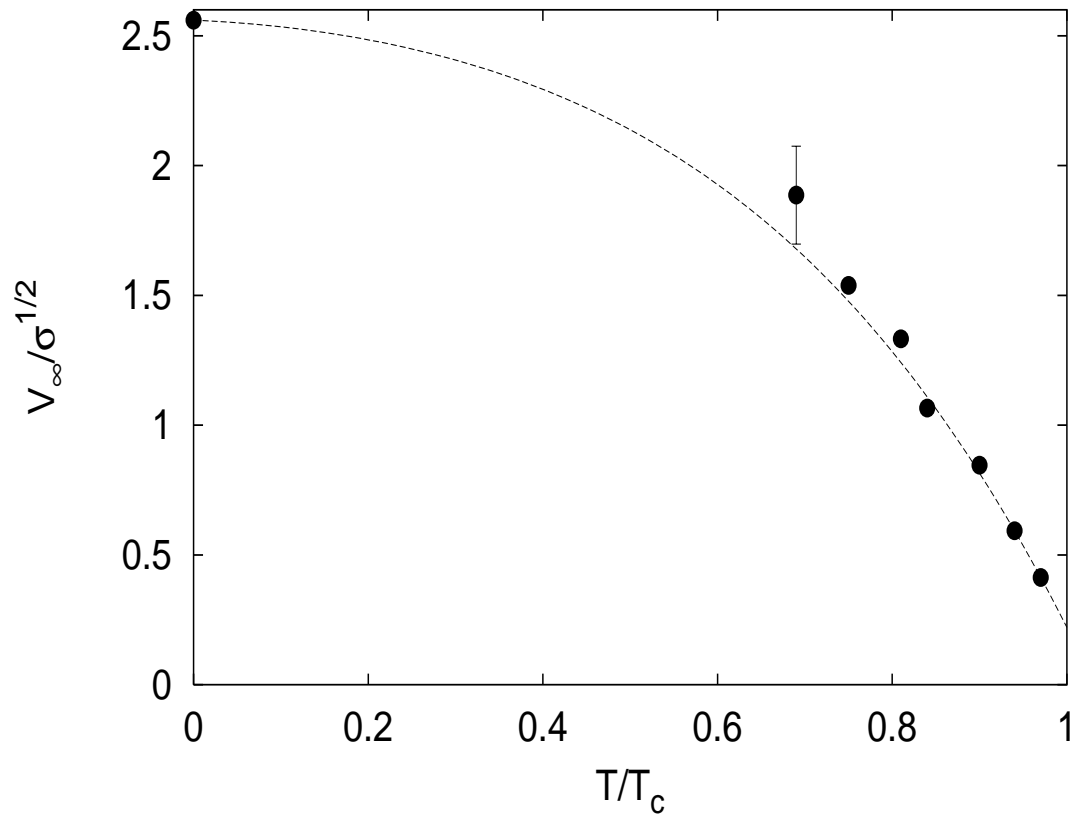

Figure 2: The temperature-dependence of the string-breaking plateau of the heavy quark potential; the zero temperature point is obtained from Eqs. (6.17). 


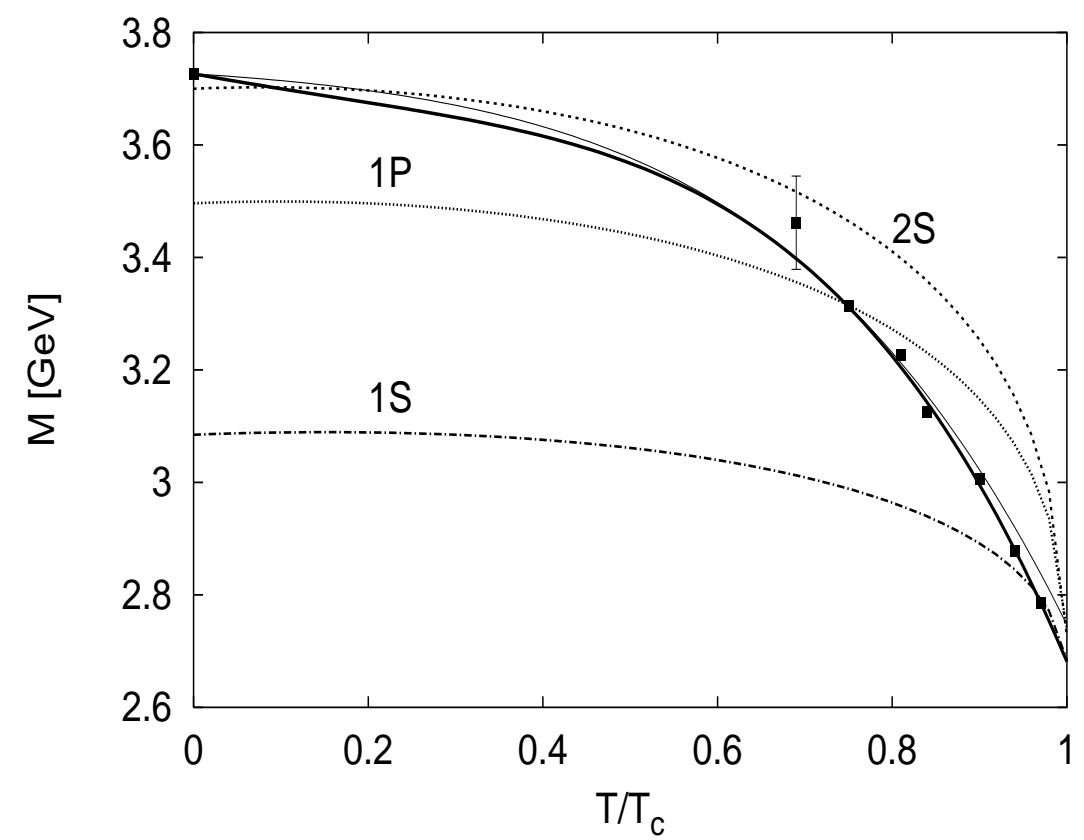

Figure 3: The open charm threshold and the masses of different charmonium states versus temperature. The thick solid line is the open charm threshold obtained by normalizing at $r T=1 / 4$, the thin solid line at $r T=\sqrt{2} / 4$.

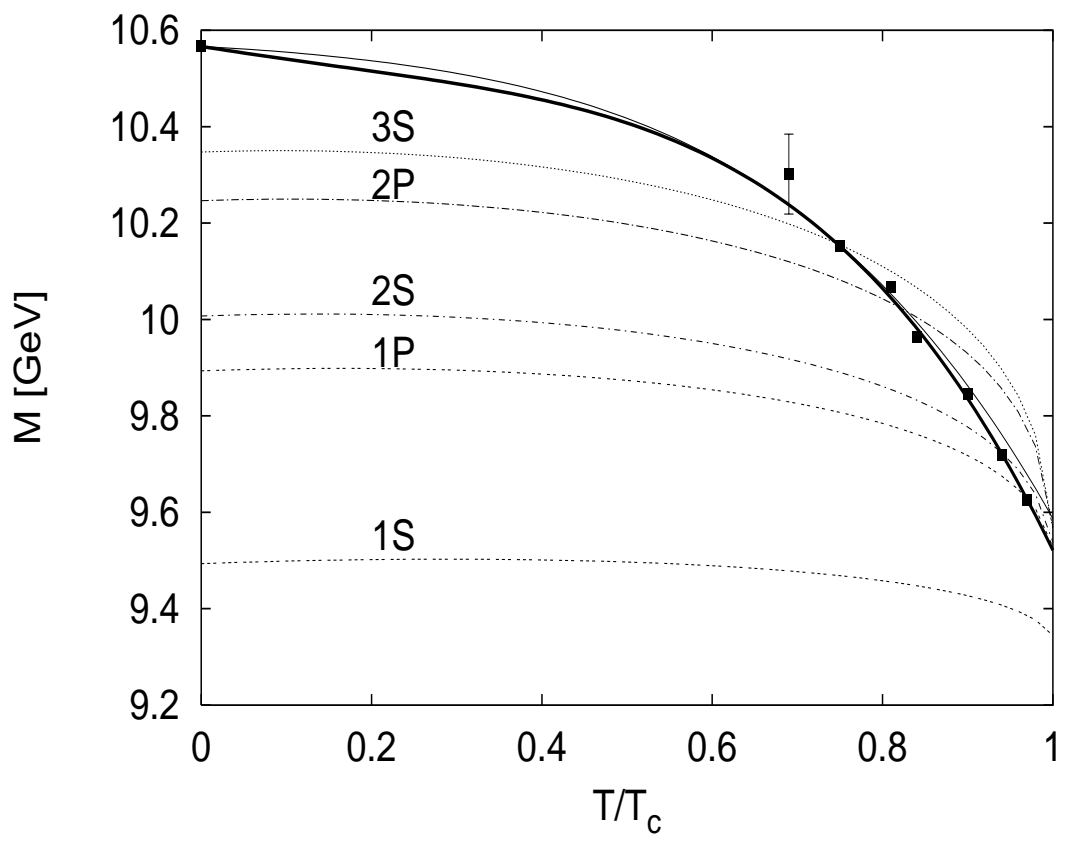

Figure 4: The open beauty threshold and the masses of different bottomonium states versus the temperature. The thick solid line is the open beauty threshold obtained by normalizing at $r T=1 / 4$, the thin solid line at $r T=\sqrt{2} / 4$. 